\title{
Value distribution of meromorphic solutions of some second order equations
}

\author{
Yunfei $\mathrm{Du}^{\mathrm{a}}$, Zongsheng $\mathrm{Gao}^{\mathrm{a}}$, Jilong Zhang ${ }^{\mathrm{a}}$, Ming Zhao ${ }^{\mathrm{b}, *}$ \\ ${ }^{a}$ LMIB-School of Mathematics and Systems Science, Beihang University, Beijing, 100191, China \\ b School of Science, China University of Geosciences, Beijing, 100083, China
}

*Corresponding author, e-mail: zhaoming311@163.com

Received 12 Mar 2018

Accepted 16 Jun 2018

\begin{abstract}
We investigate the value distribution of some second-order differential-difference and q-difference equations. In particular, the existence of Borel exceptional values, and the relationship between the exponent of convergence of zeros, fixed points, and the growth order of the transcendental meromorphic solutions of these equations are studied. The existence of the rational solutions and some other properties of these equations are also studied.
\end{abstract}

KEYWORDS: differential-difference, $q$-difference, Painlevé equation

MSC2010: 30D35 34A20

\section{INTRODUCTION}

Here we assume the reader is familiar with the basic notions of Nevanlinna theory ${ }^{1-3}$. We use $\rho(f)$ to denote the order of growth of the meromorphic function $f(z)$, and $\lambda(f)$ to denote the exponent of convergence of the zeros of $f(z)$. We also use $\tau(f)$ to denote the convergence of fixed points of $f(z)$, which is defined as $\tau(f)=$ $\limsup r \rightarrow \infty \log N(r, 1 /(f-z)) / \log r$. We also define $\bar{f} \equiv f(z+1)$ and $f \equiv f(z-1)$. Any quantity satisfying $S(r, f)=o(\bar{T}(r, f))(r \rightarrow \infty)$, possibly outside a set with finite logarithmic measure, is denoted by $S(r, f)$ and the field of small functions with respect to $f$ is denoted by $S(f)=\{\alpha$ meromorphic : $T(r, \alpha)=S(r, f)\}$.

A meromorphic solution $f$ of a difference equation is called admissible if all the coefficients of the equation are in $S(f)$. In particular, if the coefficients are rational, then an admissible solution must be transcendental, and if an admissible solution is rational, then the coefficients must be constants.

An ordinary differential equation is said to possess the Painlevé property if all of its solutions are single-values about all movable singularities ${ }^{4}$. Painlevé ${ }^{5,6}$, Fuchs ${ }^{7}$ and Gambier ${ }^{8}$ completed a substantial classification work, which comprised of sieving through a large class of second-order differential equations by making use of a criterion proposed by Picard ${ }^{9}$, now known as the Painlevé property. Painlevé and his colleagues discovered six new equations, later named the Painlevé equations, which were not solvable in terms of known functions. Actually, the Painlevé equations are six nonlinear ordinary differential equations denoted traditionally by $P_{\mathrm{I}}, P_{\mathrm{II}}, \ldots, P_{\mathrm{VI}}$.

As for the difference type Painlevé equation, it is an important topic in Nevanlinna theory. Ablowitz, Halburd, and Herbst ${ }^{10}$ studied the Painlevé difference equation $\bar{f} \star f=R(z, f)$ where $R$ is rational in both of its arguments, $\star$ stands for either addition or multiplication. They proved that the existence of a nonrational meromorphic solution of finite order

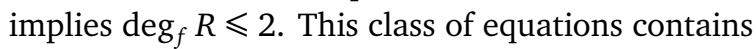
many integrable equations that are called difference Painlevé I-III equations.

Halburd and Korhonen ${ }^{11}$ considered

$$
\bar{f}+\underline{f}=R(z, f),
$$

where $R(z, f)$ is rational in $f$ and meromorphic in $z$. They proved the following theorem.

Theorem 1 (Ref. 11) If (1) has an admissible meromorphic solution of finite order, then either $f$ satisfies a difference Riccati equation

$$
f(z+1)=\frac{p(z+1) f(z)+q(z)}{f(z)+p(z)},
$$

where $p, q \in S(f)$, or equation (1) can be transformed by a linear change in $f$ to one of the following equations:

$$
f(z+1)+f(z)+f(z-1)=\frac{\pi_{1} z+\pi_{2}}{f(z)}+\kappa_{1},
$$




$$
\begin{gathered}
f(z+1)-f(z)+f(z-1)=\frac{\pi_{1} z+\pi_{2}}{f(z)}+(-1)^{z} \kappa_{1}, \\
f(z+1)+f(z-1)=\frac{\pi_{1} z+\pi_{3}}{f(z)}+\pi_{2}, \\
f(z+1)+f(z-1)=\frac{\pi_{1} z+\kappa_{1}}{f(z)}+\frac{\pi_{2}}{f^{2}(z)}, \\
f(z+1)+f(z-1)=\frac{\left(\pi_{1} z+\kappa_{1}\right) f(z)+\pi_{2}}{(-1)^{-z}-f^{2}(z)}, \\
f(z+1)+f(z-1)=\frac{\left(\pi_{1} z+\kappa_{1}\right) f(z)+\pi_{2}}{1-f^{2}(z)}, \\
f(z+1)+f(z-1)=\frac{p(z)}{f(z)} \\
f(z+1)+f(z-1)=p(z) f(z)+q(z),
\end{gathered}
$$

where $\pi_{k}, \kappa_{k} \in S(f)$ are arbitrary finite-order periodic functions with period $k$.

Equations (3), (5), and (6) are known integrable discretizations of the Painlevé I equation, while equation (8) is often referred to as the difference Painlevé II equation. Eq. (2) is a difference Riccati equation and (10) is a linear difference equation. The work on the family $f(z+1) f(z-1)=R(z, f)$, which includes the so-called difference Painlevé III equation, was initiated in Ref. 12.

Painlevé equations are a fascinating subject in mathematics. They possess many special features ${ }^{13}$. One of them is that, given a solution of a Painlevé equation $\left(P_{\mathrm{II}}, \ldots, P_{\mathrm{VI}}\right)$ with a choice of some parameter, a special method based on Bäcklund transformations can be used for deriving a new solution with a different value of the parameter, either for the same Painlevé equation or for another. Symmetry is a word used frequently to refer to such a mechanism to construct new solutions by transformations. Specially, Painlevé equations appear in many applications and fields such as hydrodynamics, plasma physics, nonlinear optics, and solid state physics.

Recently, many scholars ${ }^{14-17}$ have focused on complex differential and difference equations and given many results in value distribution theory of meromorphic functions. The aim of this paper is to investigate the properties of the differentialdifference and $q$-difference type Painlevé III equations.

Chen and Shon ${ }^{18}$ studied some of the Painlevé I and II equations, and obtained the following result.

Theorem 2 Let $a, b, c$ be constants with $a c \neq 0$. If $f(z)$ is a finite order transcendental meromorphic solution of the difference Painlevé II equation

$$
\bar{f}+\underline{f}=\frac{(a z+b) f+c}{1-f^{2}},
$$

then

(i) $f$ has at most one non-zero finite Borel exceptional value;

(ii) $\lambda(1 / f)=\lambda(f)=\rho(f)$;

(iii) $f(z)$ has infinitely many fixed points and satisfies $\tau(f)=\rho(f)$.

In the same paper, they also investigated the properties of rational solutions of equation (11) and obtained the following result.

Theorem 3 Let $a, b, c$ be constants, $a c \neq 0$. Suppose that a rational function

$$
f(z)=\frac{P(z)}{Q(z)}=\frac{p z^{m}+p_{m-1} z^{m-1}+\cdots+p_{0}}{q z^{n}+q_{n-1} z^{n-1}+\cdots+q_{0}}
$$

is a solution of (11), where $P(z)$ and $Q(z)$ are relatively prime polynomials, $p, p_{m-1}, \ldots, p_{0}$ and $q$, $q_{n-1}, \ldots, q_{0}$ are constants. Then $n=m+1$ and $p=$ $-(c / a) q$.

Zhang and $\mathrm{Yi}^{19}$ investigated the Painlevé III equations and in particular

$$
\begin{gathered}
\bar{f} \underline{f}(f-1)=\eta(f-\mu), \\
\bar{f} \underline{f}(f-1)=f^{2}-\mu f,
\end{gathered}
$$

and obtained the following theorems.

Theorem 4 (Ref. 19) If $f$ is a transcendental finiteorder meromorphic solution of (12), then

(i) $\lambda(f)=\tau(f)=\rho(f)$;

(ii) if $\mu=0$, then $f$ has at most one non-zero Borel exceptional value for $\rho(f)>0$.

Theorem 5 (Ref. 19) If $f$ is a transcendental meromorphic solution of (13) with finite order $\rho(f)>0$, then

(i) $\tau(f)=\rho(f)$;

(ii) if $\mu \neq 0$, then $\lambda(f)=\rho(f)$;

(iii) $f$ has at most one non-zero Borel exceptional value.

\section{RESULTS}

In the following, we combine differential and difference equation ideas to study the Painlevé equations, which is a new method to research this topic. We investigate some properties of meromorphic solutions and rational solutions of the two differentialdifference equations

$$
\begin{aligned}
(\bar{f} \underline{f})^{\prime} & =\frac{\eta(f-\mu)}{f-1}, \\
\bar{f}^{\prime} \underline{f}^{\prime} & =\frac{f^{2}-\mu f}{f-1},
\end{aligned}
$$

and prove the following results. 
Theorem 6 Let $\eta \neq 0, \mu \neq 1$ be constants. If $f(z)$ is a finite order transcendental meromorphic solution of equation (14), then

(i) $\tau(f)=\rho(f)$;

(ii) if $\mu \neq 0$, then $\lambda(f)=\rho(f)$;

(iii) $f$ has at most one finite Borel exceptional value.

Theorem 7 Let $\mu, \eta$ be non-zero constants. If

$$
f(z)=\frac{P(z)}{Q(z)}=\frac{a z^{p}+a_{p-1} z^{p-1}+\cdots+a_{0}}{b z^{q}+b_{q-1} z^{q-1}+\cdots+b_{0}}
$$

is a rational solution of equation (14), where $P(z)$ and $Q(z)$ are relatively prime polynomials, $a \neq 0$, $a_{p-1}, \ldots, a_{0}$ and $b \neq 0, b_{q-1}, \ldots, b_{0}$ are constants, then $p=q$ and $a / b=\mu$.

Theorem 8 Let $\mu \neq 1$ be a constant. If $f(z)$ is a finite order transcendental meromorphic solution of equation (15), then

(i) $\tau(f)=\lambda(1 / f)=\rho(f)$;

(ii) if $\mu \neq 0$, then $\lambda(f)=\rho(f)$;

(iii) if $\mu \neq 0$, then $f$ has at most one Borel exceptional value.

Theorem 9 Let $\mu \neq 0$ be a constant. If $f(z)=$ $P(z) / Q(z)$ defined by (16) is a rational solution of equation (15), then one of following holds:

(i) $p=q$ and $a / b=\mu$;

(ii) $p=q+2$ and $4 a=b$.

Then we investigate the $q$-difference equation

$$
f(q z) f(z / q)=\frac{P(z, f(z))}{Q(z, f(z))}=\frac{f(z)^{2}-\delta z^{2 \mu}}{f(z)^{2}-1},
$$

where $P(z, f(z))$ and $Q(z, f(z))$ are polynomials in $f(z)$ without common factors, and we prove the following results.

Theorem 10 Let $f(z)$ be a zero-order transcendental meromorphic solution of (17), and $\delta, \mu$ be two constants. Then

(i) $f(z)$ has infinitely many poles;

(ii) if $\delta \neq 0$ and $\mu \neq 0$, then $f(z)$ assumes every finite value infinitely often;

(iii) if $\mu=0$ and $f$ assumes a finite value $A$ finitely often, then $A$ is a solution of $z^{4}-2 z^{2}+\delta=0$.

Suppose that $f(z)$ is a rational function with poles of $z_{1}, \ldots, z_{m}$. Then $f(z)$ can be represented as the following form

$$
\begin{aligned}
f(z)=\sum_{i=1}^{m}\left(\frac{c_{i s_{1}}}{\left(z-z_{i}\right)}\right. & \left.+\cdots+\frac{c_{i s_{i}}}{\left(z-z_{i}\right)^{s_{i}}}\right) \\
& +C+C_{1} z+\cdots+C_{n} z^{n} .
\end{aligned}
$$

Theorem 11 Let $\delta$ and $\mu$ be two constants. Then

(i) if $\delta \neq 0$ and $\mu \neq 0$, suppose (18) is a rational solution of (17), then $\mu=2 n$ and $\delta=-\left(C_{n}\right)^{4}$;

(ii) if $\delta \neq 0$ and $\mu=0$, then the rational solutions of equation (17) must satisfy $f(z)=C+P(z) / Q(z)$, where $P(z)$ and $Q(z)$ are relatively prime polynomials with $\operatorname{deg} P<\operatorname{deg} Q$ and $C^{4}-2 C^{2}+\delta=0$;

(iii) if $\delta=0$, then the rational solutions of (17) must satisfy $f(z)=P(z) / Q(z)$ or $f(z)= \pm \sqrt{2}+$ $P(z) / Q(z)$, where $P(z)$ and $Q(z)$ are relatively prime polynomials with $\operatorname{deg} P<\operatorname{deg} Q$.

\section{LEMMAS}

We introduce some lemmas for the proofs of our theorems in this section.

Lemma 1 (Ref. 3) Let $f_{j}(z)(j=1, \ldots, n)(n \geqslant 2)$ be meromorphic functions, and $g_{j}(z)(j=1, \ldots, n)$ be entire functions. If

(i) $\sum_{j=1}^{n} f_{j}(z) \mathrm{e}^{g_{j}(z)} \equiv 0$;

(ii) $g_{h}(z)-g_{k}(z)$ is not a constant for $1 \leqslant h<k \leqslant n$;

(iii) $T\left(r, f_{j}(z)\right)=S\left(r, \mathrm{e}^{g_{h}(z)-g_{k}(z)}\right)$ for $1 \leqslant j \leqslant n$ and $1 \leqslant h<k \leqslant n$,

then $f_{j}(z) \equiv 0(j=1, \ldots, n)$.

Lemma 2 (Ref. 2) Let $f(z)$ be a meromorphic function. Then

$$
m\left(r, \frac{f^{\prime}}{f}\right)=S(r, f) .
$$

Remark 1 Chiang, Feng ${ }^{20}$ and Halburd, Korhonen ${ }^{21}$ proved the difference analogue of the logarithmic derivative lemma, i.e., if $f(z)$ is a finite order function and $c$ is a non-zero complex constant, then

$$
m\left(r, \frac{f(z+c)}{f(z)}\right)+m\left(r, \frac{f(z)}{f(z+c)}\right)=S(r, f) .
$$

Lemma 3 (Ref. 22) Let $f$ be a transcendental meromorphic solution of finite order $\rho$ of a difference equation of the form

$$
U(z, f) P(z, f)=Q(z, f),
$$

where $U(z, f), P(z, f)$, and $Q(z, f)$ are difference polynomials such that the total degree $\operatorname{deg}_{f} U(z, f)=$ $n$ in $f(z)$ and its shifts, and $\operatorname{deg}_{f} Q(z, f) \leqslant n$. If $U(z, f)$ contains just one term of maximal total degree in $f(z)$ and its shifts, then, for each $\varepsilon>0$,

$$
m(r, P(z, f))=O\left(r^{\rho-1+\varepsilon}\right),
$$

possibly outside an exceptional set of finite logarithmic measure. 
Remark 2 In Lemma 3, if $f(z)$ is a transcendental function with $\rho(f)<\infty$, and $U(z, f), P(z, f)$, $Q(z, f)$ are differential-difference polynomials in $f$, then by using a similar method to that in the proof of Lemma 2.4.2 of Ref. 2, we see that the similar conclusion of Lemma 3 holds.

Remark 3 When $U(z, f), P(z, f)$, and $Q(z, f)$ are $q$ difference polynomials in a non-constant zero-order meromorphic function $f$, we can also obtain the same conclusion as in Lemma $3^{15}$.

Lemma 4 (Ref. 23) Let $f(z)$ be a transcendental meromorphic solution of finite order of the equation $P(z, f)=0$, where $P(z, f)$ is a differential-difference polynomial in $f(z)$. If $P(z, a) \not \equiv 0$ for a meromorphic function $a \in S(f)$, then

$$
m\left(r, \frac{1}{f-a}\right)=S(r, f)
$$

outside of a possible exceptional set of finite logarithmic measure.

Remark 4 When $P(z, f)$ are $q$-difference polynomials in non-constant zero-order meromorphic $f$, it has the same conclusion as in Lemma $4^{15}$.

Lemma 5 (Ref. 24) Let $f(z)$ be a zero-order meromorphic function, and $q \in C \backslash\{0\}$. Then

$$
\begin{gathered}
T(r, f(q z))=(1+o(1)) T(r, f(z)), \\
N(r, f(q z))=(1+o(1)) N(r, f(z))
\end{gathered}
$$

on a set of lower logarithmic density 1.

\section{PROOFS OF THEOREMS}

Proof of Theorem 6: (i) Denote $g(z)=f(z)-z$. Since $f(z)$ is transcendental, we obtain $T(r, g)=$ $T(r, f)+S(r, f)$. Substituting $f(z)=g(z)+z$ into (14) yields

$$
[(\bar{g}+z+1)(\underline{g}+z-1)]^{\prime}=\frac{\eta(g+z-\mu)}{g+z-1} .
$$

Then we have

$$
\begin{array}{r}
P(z, g)=[(\bar{g}+z+1)(\underline{g}+z-1)]^{\prime}(g+z-1) \\
-\eta(g+z-\mu)=0 .
\end{array}
$$

It follows from (19) that

$$
P(z, 0)=2 z^{2}-(\eta+2) z+\eta \mu \not \equiv 0 .
$$

Combining this with Lemma 4 yields

$$
m\left(r, \frac{1}{g}\right)=S(r, f)=m\left(r, \frac{1}{f-z}\right) .
$$

Thus

$$
N\left(r, \frac{1}{f-z}\right)=T(r, f)+S(r, f),
$$

which means $\tau(f)=\rho(f)$.

(ii) If $\mu \neq 0$, we denote

$$
Q(z, f):=(\bar{f} \underline{f})^{\prime}(f-1)-\eta(f-\mu)=0 .
$$

Then $Q(z, 0)=\eta \mu \neq 0$. Combining this with Lemma 4 yields $m(r, 1 / f)=S(r, f)$, i.e., $N(r, 1 / f)=$ $T(r, f)+S(r, f)$, which implies $\lambda(f)=\rho(f)$.

(iii) Assume that $f$ has two finite Borel exceptional values $a$ and $b(\neq a)$. Set

$$
g(z)=\frac{f(z)-a}{f(z)-b} .
$$

Then

$$
\begin{aligned}
\rho(g) & =\rho(f), \\
\lambda(g) & =\lambda(f-a)<\rho(g), \\
\lambda\left(\frac{1}{g}\right) & =\lambda(f-b)<\rho(g) .
\end{aligned}
$$

Since $g$ is of finite order, we write it as

$$
g(z)=h(z) \mathrm{e}^{\mathrm{d} z^{n}},
$$

where $d \neq 0$ is a constant, $n \geqslant 1$ is an integer, and $h(z)$ is a meromorphic function which satisfies

$$
\rho(h)<\rho(g)=n .
$$

It follows from (20) and (21) that

$$
f(z)=\frac{a-b g(z)}{1-g(z)}=\frac{a-b h(z) \mathrm{e}^{d z^{n}}}{1-h(z) \mathrm{e}^{d z^{n}}}
$$

and

$$
\bar{f}=\frac{a-b \bar{h} h_{1}(z) \mathrm{e}^{d z^{n}}}{1-\bar{h} h_{1}(z) \mathrm{e}^{d z^{n}}}, \underline{f}=\frac{a-b \underline{h} h_{2}(z) \mathrm{e}^{d z^{n}}}{1-\underline{h} h_{2}(z) \mathrm{e}^{d z^{n}}},
$$

where $h_{1}(z)=\exp \left(n d z^{n-1}+\cdots+d\right)$ and $h_{2}(z)=$ $\exp \left(-n d z^{n-1}+\cdots+(-1)^{n} d\right)$. Substituting (22) and (23) into (14) yields

$$
\begin{aligned}
A(z) \mathrm{e}^{5 \mathrm{~d} z^{n}} & +B(z) \mathrm{e}^{4 \mathrm{~d} z^{n}}+C(z) \mathrm{e}^{3 \mathrm{~d} z^{n}} \\
& +D(z) \mathrm{e}^{2 \mathrm{~d} z^{n}}+E(z) \mathrm{e}^{\mathrm{d} z^{n}}+F(z)=0,
\end{aligned}
$$


where $A(z)=\eta(\mu-b) h\left(\bar{h} \underline{h} h_{1} h_{2}\right)^{2}$,

$$
\begin{aligned}
& B(z)= b(a-b)(b-1) h\left[m_{1}\left(\underline{h} h_{2}\right)^{2}+m_{2}\left(\bar{h} h_{1}\right)^{2}\right] \\
&+2 \eta(b-\mu) h \bar{h} \underline{h} h_{1} h_{2}\left(\bar{h} h_{1}+\underline{h} h_{2}\right) \\
&+\eta(a-\mu)\left(\bar{h} \underline{h} h_{1} h_{2}\right)^{2},
\end{aligned}
$$

$$
\begin{gathered}
C(z)=\eta(\mu-b) h\left[\left(\bar{h} h_{1}+\underline{h} h_{2}\right)^{2}+2 \bar{h} \underline{h} h_{1} h_{2}\right] \\
+b(a-1)(b-a)\left[m_{1}\left(\underline{h} h_{2}\right)^{2}+m_{2}\left(\bar{h} h_{1}\right)^{2}\right] \\
+(b-1)\left(b^{2}-a^{2}\right) h\left(m_{1} \underline{h} h_{2}+m_{2} \bar{h} h_{1}\right) \\
+2 \eta(\mu-a) \bar{h} \underline{h} h_{1} h_{2}\left(\bar{h} h_{1}+\underline{h} h_{2}\right),
\end{gathered}
$$

$$
\begin{aligned}
D(z)= & \eta(a-\mu)\left[\left(\bar{h} h_{1}+\underline{h} h_{2}\right)^{2}+2 \bar{h} \underline{h} h_{1} h_{2}\right] \\
+ & \left(a^{2}-b^{2}\right)(a-1)\left(m_{2} \bar{h} h_{1}+m_{1} \underline{h} h_{2}\right) \\
& +a(a-b)(b-1) h\left(m_{1}+m_{2}\right) \\
& +2 \eta(b-\mu) h\left(\bar{h} h_{1}+\underline{h} h_{2}\right),
\end{aligned}
$$

$$
\begin{aligned}
E(z)=\eta(\mu-b) h- & 2 \eta(a-\mu)\left(\bar{h} h_{1}+\underline{h} h_{2}\right) \\
& -a(a-b)(a-1)\left(m_{1}+m_{2}\right),
\end{aligned}
$$

$F(z)=\eta(a-\mu)$, where

$$
m_{1}=\bar{h}^{\prime} h_{1}+\bar{h} h_{1}^{\prime}+n d z^{n-1} \bar{h} h_{1}
$$

and

$$
m_{2}=\underline{h}^{\prime} h_{2}+\underline{h} h_{2}^{\prime}+n d z^{n-1} \underline{h} h_{2} .
$$

From Lemma 1 and (24), we have

$$
A(z) \equiv B(z) \equiv C(z) \equiv D(z) \equiv E(z) \equiv F(z) \equiv 0 .
$$

Since $\eta \neq 0$, it follows from $A(z) \equiv F(z) \equiv 0$ that $b=\mu=a$, which is a contradiction. Hence $f$ has at most one finite Borel exceptional value.

Proof of Theorem 7: Substituting $f(z)=P(z) / Q(z)$ into (14) yields

$$
\left(\frac{\bar{P}}{\bar{Q}} \frac{P}{\bar{Q}}\right)^{\prime}\left(\frac{P(z)}{Q(z)}-1\right)=\eta\left(\frac{P(z)}{Q(z)}-\mu\right) .
$$

Let $s=p-q$. We consider three cases.

Case 1: $s>0$. Then $P(z) / Q(z)=(a / b) z^{s}(1+$ $o(1))$ as $r \rightarrow \infty$. Substituting it into (25), we obtain

$$
\begin{aligned}
2 s\left(\frac{a}{b}\right)^{2} z^{2 s-1}(1+o(1)) & \left(\frac{a}{b} z^{s}(1+o(1))-1\right) \\
= & \eta\left(\frac{a}{b} z^{s}(1+o(1))-\mu\right),
\end{aligned}
$$

which is a contradiction as $r \rightarrow \infty$.
Case 2: $s<0$. Then $P(z) / Q(z)=o(1), \bar{P} / \bar{Q}=$ $o(1)$, and $\underline{P} / \underline{Q}=o(1)$ as $r \rightarrow \infty$. It follows from (25) that $\mu=0$. Then (25) can be rewritten as

$$
\begin{array}{r}
{\left[\left(\bar{P}^{\prime} \underline{P}+\bar{P} \underline{P}^{\prime}\right) \bar{Q} \underline{Q}-\left(\bar{Q}^{\prime} \underline{Q}+\bar{Q} \underline{Q^{\prime}}\right) \bar{P} \underline{P}\right](P(z)-Q(z))} \\
=\eta P(z)(\bar{Q} \underline{Q})^{2} .
\end{array}
$$

Observing the above equation, we see the degrees of both sides are not the same, so (14) does not have rational solution under this condition.

Case 3: $s=0$. We have $P(z) / Q(z)=a / b+o(1)$ as $r \rightarrow \infty$. Combining this with (25) yields $a / b=$ $\mu$.

Proof of Theorem 8: (i) Let $\phi(z)=f(z)-z$. Since $f(z)$ is a transcendental function, we have $T(r, \phi)=$ $T(r, f)+S(r, f)$. Then, substituting $f(z)=\phi(z)+z$ into (15), we obtain

$(\bar{\phi}+z+1)^{\prime}(\underline{\phi}+z-1)^{\prime}(\phi+z-1)=(\phi+z)^{2}-\mu(\phi+z)$.

Let

$$
\begin{array}{r}
P(z, \phi)=(\bar{\phi}+z+1)^{\prime}(\underline{\phi}+z-1)^{\prime}(\phi+z-1) \\
-(\phi+z)^{2}+\mu(\phi+z) .
\end{array}
$$

Then

$$
P(z, 0)=-z^{2}+(\mu+1) z-1 \not \equiv 0,
$$

and by Lemma 4 we have

$$
m\left(r, \frac{1}{f-z}\right)=m\left(r, \frac{1}{\phi}\right)=S(r, \phi)=S(r, f)
$$

outside of a possible exceptional set of finite logarithmic measure, which means $N(r, 1 /(f-z))=$ $T(r, f)+S(r, f)$. Hence we have $\tau(f)=\rho(f)$. We rewrite equation (15) as

$$
\left((\bar{f})^{\prime}(\underline{f})^{\prime}-f\right) f=(\bar{f})^{\prime}(\underline{f})^{\prime}-\mu f .
$$

It follows from Lemma 4 and Remark 2 that $m(r, f)=S(r, f)$, which means $N(r, f)=T(r, f)+$ $S(r, f)$, i.e., $\lambda(1 / f)=\rho(f)$.

(ii) If $\mu \neq 0$, we rewrite (26) as

$$
\frac{1}{f}=-\frac{1}{\mu} \frac{\bar{f}}{\bar{f}} \overline{\bar{f}}\left(\frac{(\bar{f})^{\prime}}{\bar{f}} \frac{(f)^{\prime}}{\underline{f}} f-\frac{(\bar{f})^{\prime}}{\bar{f}} \frac{(f)^{\prime}}{\underline{f}}-\frac{f}{\bar{f}} \frac{f}{\underline{f}}\right) .
$$

Combining Lemma 2 and Remark 2 yields

$$
m\left(r, \frac{1}{f}\right)=m(r, f)+S(r, f)=S(r, f) .
$$

Thus $\lambda(f)=\rho(f)$ holds. 
(iii) If $\mu \neq 0$, by (i) and (ii), we see that $0, \infty$ must not be the Borel exceptional values of $f(z)$. Then assume that $f(z)$ has two non-zero finite Borel exceptional values $a$ and $b(\neq a)$. We set

$$
g(z)=\frac{f(z)-a}{f(z)-b} .
$$

Then $\rho(g)=\rho(f), \lambda(g)=\lambda(f-a)<\rho(f)=\rho(g)$, and $\lambda(1 / g)=\lambda(f-b)<\rho(f)=\rho(g)$. Since $g(z)$ is of finite order, we rewrite it as

$$
g(z)=h(z) \mathrm{e}^{\mathrm{d} z^{n}}
$$

where $d \neq 0$ is a constant, $n \geqslant 1$ is an integer, and $h(z)$ is a meromorphic function satisfying

$$
\rho(h)<\rho(g)=n .
$$

Then

$$
\bar{g}=\bar{h} h_{1}(z) \mathrm{e}^{\mathrm{d} z^{n}}, \underline{g}=\underline{h} h_{2}(z) \mathrm{e}^{\mathrm{d} z^{n}},
$$

where

$$
h_{1}(z)=\mathrm{e}^{n d z^{n-1}+\cdots+d}
$$

and

$$
h_{2}(z)=\mathrm{e}^{-n d z^{n-1}+\cdots+(-1)^{n} d} .
$$

It follows from (27), (28), (29) and (15) that

$$
\begin{gathered}
A(z) \mathrm{e}^{6 \mathrm{~d} z^{n}}+B(z) \mathrm{e}^{5 \mathrm{~d} z^{n}}+C(z) \mathrm{e}^{4 \mathrm{~d} z^{n}}+D(z) \mathrm{e}^{3 \mathrm{~d} z^{n}} \\
+E(z) \mathrm{e}^{2 \mathrm{~d} z^{n}}+F(z) \mathrm{e}^{\mathrm{d} z^{n}}+G(z)=0,
\end{gathered}
$$

where

$$
\begin{gathered}
A(z)=b(b-\mu)\left(h \bar{h} \underline{h} h_{1} h_{2}\right)^{2} \\
B(z)=\left[(\mu(a+b)-2 a b) \bar{h} \underline{h} h_{1} h_{2}\right. \\
\left.-2 b(b-\mu) h\left(\bar{h} h_{1}+\underline{h} h_{2}\right)\right] h \bar{h} \underline{h} h_{1} h_{2} \\
C(z)=\left[(b-\mu) h\left(b h+2 a \bar{h} h_{1}\right)+(a-\mu)\left(a \bar{h} h_{1}\right.\right. \\
\left.+2 b h) \bar{h} h_{1}\right]\left(\underline{h} h_{2}\right)^{2}-(a-b)^{2}(b-1) h^{2} m_{1} m_{2} \\
-2\left[(\mu(a+b)-2 a b) \bar{h} h_{1}-2 b(b-\mu) h\right] h \bar{h} \underline{h} h_{1} h_{2} \\
+b(b-\mu)\left(h \bar{h} h_{1}\right)^{2}, \\
D(z)=\left[(\mu a+\mu b-2 a b) \bar{h} h_{1}-2 b(b-\mu) h\right] h \bar{h} h_{1} \\
+(\mu a+\mu b-2 a b) h-2 a(a-\mu) \bar{h} h_{1} \\
\left.+(a-\mu)\left(a \bar{h} h_{1}+2 b h\right) \bar{h} h_{1}\right] \underline{h} h_{2} \\
-2\left[(b-\mu) h\left(b h+2 a \bar{h} h_{1}\right)\right. \\
-(a-b)^{3} h m_{1} m_{2},
\end{gathered}
$$

$$
\begin{gathered}
E(z)=a(a-\mu)\left(\underline{h} h_{2}\right)^{2}+(a-\mu)\left(a \bar{h} h_{1}+2 b h\right) \bar{h} h_{1} \\
-2\left[(\mu a+\mu b-2 a b) h-2 a(a-\mu) \bar{h} h_{1}\right] \underline{h} h_{2} \\
\quad+(b-\mu)\left(b h^{2}+2 a h \bar{h} h_{1}\right) \\
\quad-(a-1)(a-b)^{2} m_{1} m_{2}, \\
F(z)=(\mu a+\mu b-2 a b) h-2 a(a-\mu)\left(\bar{h} h_{1}+\underline{h} h_{2}\right), \\
G(z)=a(a-\mu),
\end{gathered}
$$

where

$$
m_{1}=\bar{h}^{\prime} h_{1}+\bar{h} h_{1}^{\prime}+n d z^{n-1} \bar{h} h_{1}
$$

and

$$
m_{2}=\underline{h}^{\prime} h_{2}+\underline{h} h_{2}^{\prime}+n d z^{n-1} \underline{h} h_{2} .
$$

Combining Lemma 1 and (30) yields

$$
\begin{aligned}
A(z) & \equiv B(z) \equiv C(z) \equiv D(z) \equiv E(z) \\
& \equiv F(z) \equiv G(z) \equiv 0 .
\end{aligned}
$$

Since $a$ and $b$ are non-zero constants, we deduce from $A(z) \equiv 0$ and $G(z) \equiv 0$ that $a(a-\mu)=0$ and $b(b-\mu)=0$ which means that $a$ and $b$ are distinct non-zero roots of the equation $z(z-\mu)=0$, so $a=$ $\mu=b$, which is a contradiction, i.e., $f(z)$ cannot have two Borel exceptional values.

Proof of Theorem 9: Substituting $f(z)=P(z) / Q(z)$ into (15), we have

$$
\left(\frac{\bar{P}}{\bar{Q}}\right)^{\prime}\left(\frac{P}{\bar{Q}}\right)^{\prime}\left(\frac{P(z)}{Q(z)}-1\right)=\left(\frac{P(z)}{Q(z)}\right)^{2}-\mu \frac{P(z)}{Q(z)} .
$$

Set $s=p-q$. We consider the three cases.

Case 1: $s>0$. Then $P(z) / Q(z)=(a / b) z^{s}(1+$ $o(1))$ as $r \rightarrow \infty$. Substituting it into (31) yields

$$
\begin{gathered}
s^{2}\left(\frac{a}{b}\right)^{2} z^{2(s-1)}(1+o(1))\left(\frac{a}{b} z^{s}(1+o(1))-1\right) \\
=\left(\left(\frac{a}{b}\right)^{2} z^{2 s}-\mu \frac{a}{b} z^{s}\right)(1+o(1))
\end{gathered}
$$

as $r \rightarrow \infty$. If (32) holds, then $s=2$ and $4 a=b$.

Case 2: $s<0$. It follows from (31) that

$$
\begin{aligned}
\left(\bar{P}^{\prime} \bar{Q}-\bar{Q}^{\prime} \bar{P}\right)\left(\underline{P}^{\prime} \underline{Q}-\underline{P} \underline{Q}^{\prime}\right)\left(P Q-Q^{2}\right) \\
=\left(P^{2}-\mu P Q\right)(\bar{Q} \underline{Q})^{2} .
\end{aligned}
$$

Comparing the degrees of both sides of (33), we obtain a contradiction. So under this condition, equation (15) has no rational solution.

Case 3: $s=0$. We have $P(z) / Q(z)=a / b+o(1)$ as $r \rightarrow \infty$. Combining this with (31) yields $a / b=$ $\mu$. 
Proof of Theorem 10: (i) Suppose that $f(z)$ is a zeroorder transcendental meromorphic solution of (17). Then substituting it into (17), we have

$$
f(z)^{2} f(q z) f(z / q)=f(z)^{2}+f(q z) f(z / q)-\delta z^{2 \mu} .
$$

It follows from Remark 3 that

$$
m(r, f(q z) f(z / q))=S(r, f)
$$

on a set of logarithmic density 1 . Combining this with Lemma 5 yields

$$
\begin{aligned}
N(r, f(q z) f(z / q)) & \leqslant N(r, f(q z))+N(r, f(z / q)) \\
& =2(1+o(1)) N(r, f)
\end{aligned}
$$

on a set of lower logarithmic density 1 . By the Valiron-Mohon'ko theorem ${ }^{2}$ and (17), we obtain

$$
T(r, f(q z) f(z / q))=2 T(r, f)+S(r, f) .
$$

It follows from (34), (35), and (36) that

$$
2(1+o(1)) N(r, f) \geqslant 2 T(r, f)+S(r, f)
$$

on a set of logarithmic density 1 , which means $f(z)$ has infinitely many poles.

(ii) For any finite value $A$, let $g(z)=f(z)-A$. Substituting $f(z)=g(z)+A$ into (17) yields

$$
(g(q z)+A)(g(z / q)+A)=\frac{(g(z)+A)^{2}-\delta z^{2 \mu}}{(g(z)+A)^{2}-1} .
$$

Then let

$$
\begin{gathered}
P(z, g)=(g(q z)+A)(g(z / q)+A)\left((g(z)+A)^{2}-1\right) \\
-(g(z)+A)^{2}+\delta z^{2 \mu}=0 .
\end{gathered}
$$

By the assumptions $\delta \neq 0$ and $\mu \neq 0$, we have

$$
P(z, 0)=A^{4}-2 A^{2}+\delta z^{2 \mu} \not \equiv 0 .
$$

Applying Remark 4 yields

$$
m\left(r, \frac{1}{g}\right)=S(r, f)
$$

on a set of logarithmic density 1 . That is,

$$
\begin{aligned}
N\left(r, \frac{1}{f-A}\right) & =N\left(r, \frac{1}{g}\right) \\
& =T(r, g)+S(r, g)=T(r, f)+S(r, f)
\end{aligned}
$$

on a set of logarithmic density 1 . Thus the conclusion (ii) holds. (iii) If $\mu=0$ and $A$ is not a solution of $z^{4}-2 z^{2}+$ $\delta=0$. It follows from (37) that

$$
P(z, 0)=A^{4}-2 A^{2}+\delta \neq 0 .
$$

We can obtain $N(r, 1 /(f-A))=T(r, f)+S(r, f)$ by the same method as the above argument. So $f(z)$ assumes a finite value $A$ finitely often. Then $A$ must be a solution of $z^{4}-2 z^{2}+\delta=0$.

Proof of Theorem 11: Suppose that $f(z)$ is a rational solution of (17) and has poles $z_{1}, \ldots, z_{m}$. Then $f(z)$ can be represented as

$$
\begin{aligned}
f(z)=\sum_{i=1}^{m}\left(\frac{c_{i s_{1}}}{\left(z-z_{i}\right)}\right. & \left.+\cdots+\frac{c_{i s_{i}}}{\left(z-z_{i}\right)^{s_{i}}}\right) \\
& +C+C_{1} z+\cdots+C_{n} z^{n},
\end{aligned}
$$

where $c_{i s_{i}} \neq 0, C, C_{1}, \ldots, C_{n}$ are constants. We assume $C_{n} \neq 0(n \geqslant 1)$. For sufficiently large $z$, it follows from (38) that

$$
\begin{gathered}
f(z)=C_{n} z^{n}(1+o(1)), \\
f(q z)=C_{n} z^{n} q^{n}(1+o(1)), \\
f(z / q)=C_{n} z^{n} \frac{1}{q^{n}}(1+o(1)) .
\end{gathered}
$$

From (17), we obtain

$$
\begin{aligned}
f(z)^{2} f(q z) f(z / q)=f(q z) & f(z / q) \\
& +f(z)^{2}-\delta z^{2 \mu} .
\end{aligned}
$$

Combining (39) and (40) yields

$$
\left(C_{n}\right)^{4} z^{4 n}(1+o(1))=2\left(C_{n}\right)^{2} z^{2 n}(1+o(1))-\delta z^{2 \mu} .
$$

(i) If $\delta \neq 0$ and $\mu \neq 0$, we discuss the following two cases. Case 1. If (41) holds, we have $\mu=2 n$ and $\delta=-\left(C_{n}\right)^{4}$. Case 2. If Case 1 does not hold, it follows from $C_{n} \neq 0$ that (41) is a contradiction for sufficiently large $z$. Hence $C_{1}=\cdots=C_{n}=0$. Furthermore, if $C \neq 0$, we deduce from (38) that

$$
f(q z)=f(z)=f(z / q)=C+o(1)
$$

for sufficiently large z. Substituting (42) into (40) yields

$$
C^{4}(1+o(1))=2 C^{2}(1+o(1))-\delta z^{2 \mu},
$$

which is a contradiction when $z$ is large enough. So $C=C_{1}=\cdots=C_{n}=0$. Then we have

$$
f(z)=\frac{P(z)}{Q(z)},
$$


where $P(z)$ and $Q(z)$ are polynomials such that $\operatorname{deg} P<\operatorname{deg} Q$. Substituting (43) into (17), it follows that

$$
\begin{aligned}
& P(z)^{2} P(q z) P(z / q)+\delta z^{2 \mu} Q(z)^{2} Q(q z) Q(z / q) \\
& =Q(z)^{2} P(q z) P(z / q)+P(z)^{2} Q(q z) Q(z / q) .
\end{aligned}
$$

Comparing the degrees of both sides of (44), we obtain a contradiction.

(ii) If $\delta \neq 0$ and $\mu=0$, it follows from (41) and Case 2 of (i) that $C_{1}=\cdots=C_{n}=0$. Hence

$$
f(z)=\frac{P(z)}{Q(z)}+C,
$$

where $P(z)$ and $Q(z)$ are polynomials with $\operatorname{deg} P<$ $\operatorname{deg} Q$. Furthermore, we have

$$
f(q z)=f(z)=f(z / q)=C+o(1)
$$

for sufficiently large $z$. Substituting (45) into (17) yields $C^{4}-2 C^{2}-\delta=0$.

(iii) If $\delta=0$, using the similar method as above, we have $C=0$ or $C= \pm \sqrt{2}$.

Acknowledgements: This work was supported by the National Science Foundation of China (Nos. 11171013 and 11371225) and Fundamental Research Funds for the Central Universities.

\section{REFERENCES}

1. Hayman WK (1964) Meromorphic Functions, Clarendon Press, Oxford.

2. Laine I (1993) Nevanlinna Theory and Complex Differential Equations, Walter de Gruyter, Berlin.

3. Yang CC, Yi HX (2003) Uniqueness Theory of Meromorphic Functions, Kluwer Academic, Dordrecht.

4. Gromak VI, Laine I, Shimomura S (2002) Painlevé Differential Equations in the Complex Plane, Walter de Gruyter, Berlin.

5. Painlevé P (1900) Mémoire sur les équations différentielles dont 1 intégrale générale est uniforme. Bull Soc Math Fr 28, 201-61.

6. Painlevé $P$ (1902) Sur les équations différentielles du second ordre et d ordre supérieur dont 1 intégrale générale est uniforme. Acta Math 25, 1-85.

7. Fuchs L (1905) Sur quelques équations différentielles linéares du second ordre. Compte Rendus Acad Sci Paris 141, 555-8.

8. Gambier B (1910) Sur les équations différentielles du second ordre et du premier degré dont 1 intégrale générale est á points critiques fixes. Acta Math 33, $1-55$.

9. Picard É (1889) Mémoire sur la théorie des fonctions algébriques de deux variables. $J$ Math $4 \mathrm{~V}$, 135-319.(French).
10. Ablowitz M, Halburd RG, Herbst B (2000) On the extension of Painlevé property to difference equations. Nonlinearity 13, 889-905.

11. Halburd RG, Korhonen RJ (2007) Finite-order meromorphic solutions and discrete Painlevé equtions. Proc Lond Math Soc 94, 443-74.

12. Halburd RG, Korhonen RJ (2007) Meromorphic solutions of difference equations, integrability and the discrete Painlevé equations. J Phys A: Math Gen 40, R1-R38.

13. Al-Ghassani A (2010) Measures of Growth of Discrete Rational Equations. $\mathrm{PhD}$ thesis, Loughborough Univ.

14. Zhang JL (2014) Meromorphic solutions of difference Painlevé IV equations. Adv Difference Equat 2014, 260.

15. Barnett DC, Halburd RG, Korhonen RJ, Morgan W (2007) Nevanlinna theory for the $q$-difference operator and meromorphic solutions of $q$-difference equations. Proc Roy Soc Edinb A 137, 457-74.

16. Qi XG, Yang LZ (2015) Properties of meromorphic solutions of $q$-difference equations. Electron J Differ Equat 29, 59.

17. Ronkainen O (2010) Meromorphic solutions of difference Painlevé equations. PhD thesis, Univ of Eeastern Finland.

18. Chen ZX, Shon KH (2010) Value distribution of meromorphic solutions of certain difference Painlevé equations. J Math Anal Appl 364, 556-66.

19. Zhang JL, Yi HX (2013) Properties of meromorphic solutions of Painlevé III difference equations. Adv Difference Equat 2013, 256.

20. Chiang YM, Feng SJ (2008) On the Nevanlinna characteristic of $f(z+\eta)$ and difference equations in the complex plane. Ramanujan J 16, 105-29.

21. Halburd RG, Korhonen RJ (2006) Difference analogue of the lemma on the logarithmic derivative with applications to difference equations. $J$ Math Anal Appl 314, 477-87.

22. Laine I, Yang CC (2007) Clunie theorems for difference and $q$-difference polynomials. $J$ Lond Math Soc 76, 556-66.

23. Halburd RG, Korhonen RJ (2017) Growth of meromorphic solutions of delay differential equations. Proc Am Math Soc 145, 2513-26.

24. Zhang JL, Korhonen R (2010) On the Nevanlinna characteristic of $f(q z)$ and its applications. $J$ Math Anal Appl 369, 537-44. 